\title{
Negative lift characteristics of NACA 0012 aerofoil at low Reynolds numbers
}

\author{
C PRANESH, M SIVAPRAGASAM*D, M D DESHPANDE and H K NARAHARI \\ Department of Automotive and Aeronautical Engineering, M S Ramaiah University of Applied Sciences, \\ Bengaluru 560058, India \\ e-mail: sivapragasam.aae.et@msruas.ac.in
}

MS received 17 October 2017; revised 3 May 2018; accepted 4 September 2018; published online 4 January 2019

\begin{abstract}
Numerical investigations on the flow over NACA 0012 aerofoil are carried out to provide better understanding of the unusual lift characteristics exhibited by this aerofoil at low Reynolds numbers. Computations are carried out at $R e=10,000-100,000$, for different values of angles of attack and freestream turbulence intensity. There exists a narrow range of these parameters where the net circulation around this symmetrical aerofoil is negative, leading to the generation of negative lift at positive angles of attack. Different flow regimes are identified and physical explanations are given for this unusual behaviour of negative lift, and the influence of different flow parameters is discussed.
\end{abstract}

Keywords. NACA 0012 aerofoil; low-Reynolds-number flows; flow separation; transition; laminar separation bubble; negative lift.

\section{Introduction}

The growing need for small sized flying vehicles for a variety of applications has resulted in an extensive research on Micro-Air Vehicles (MAVs). Because of their small size and slow speed, MAVs operate in a low-Reynolds-number (Re) regime $\left(10^{4}-10^{5}\right)$. At such $R e$, the aerodynamic properties of lifting surfaces are influenced by laminar flow separation, transition to turbulence, and reattachment, leading to the formation of Laminar Separation Bubble (LSB). The aim of the present work is to numerically investigate the unusual lift characteristics exhibited by NACA 0012 aerofoil at low $R e$ using a laminar-turbulent transition model. A symmetrical aerofoil at a positive angle of attack, $\alpha$, generating positive lift is well established starting from experiments, inviscid flow theories and several viscous models. However, for a given symmetrical aerofoil, there exists a narrow range of parameters of $R e, \alpha$ and freestream turbulence intensity $T u$ where the lift coefficient, $C_{l}$, is negative at positive $\alpha$. Such an observation was first made by Mueller and Batill [1] in their experimental investigation of flow around NACA $66_{3}-018$ aerofoil at $R e=130,000$. Ohtake et al [2], in their experiments with NACA 0012 aerofoil, identified a similar phenomenon in a wider $R e$ range (30,000-70,000). More recently, Tank et al [3] have also observed negative lift close to $\alpha=0^{\circ}$ at $R e=50,000$. The flow over NACA 0012

*For correspondence aerofoil in the aforementioned $R e$ range has been widely studied by various investigators; however, only a few attempts have been made to further investigate this interesting phenomenon observed at low angles of attack; see, for instance, [4, 5]. In the present article, we numerically investigate the flow over NACA 0012 aerofoil at $R e=10,000-100,000$, with varying $\alpha$ and $T u$. For a certain range of these parameters, $C_{l}$ values were negative at positive $\alpha$. Apart from quantifying these results, we provide physical explanation for the occurrence of this behaviour.

\section{Computational procedure}

The two-dimensional, incompressible, steady-state Reynolds-averaged Navier-Stokes equations were solved numerically using the commercial CFD code ANSYS Fluent. The SST $k-\omega$ model was used in conjunction with the $\gamma-R e_{\theta}$ model. A detailed description of the $\gamma-R e_{\theta}$ model is available in [6], and its successful application for several flow cases in [7]. The equations were solved with a formally second-order accurate numerical scheme. For all the computations, $y_{\max }^{+}<1$. The convergence was achieved with all residuals to be less than $10^{-7}$.

The C-shaped computational domain, with structured grid topology, extended $10 c$ ahead of and $20 c$ behind the leading edge (L.E.) of the aerofoil, where $c$ is the aerofoil chord length. Velocity inlet and pressure outlet boundary conditions were applied at domain boundaries, while the 
no-slip boundary condition was imposed on the aerofoil surface. The mesh sensitivity was evaluated using Grid Convergence Index with three increasingly fine grid resolutions, and the grid chosen for computations had 115,236 cells, since it had acceptably low discretisation error.

\section{Results and discussion}

\subsection{Aerodynamic coefficients}

The variation of lift coefficient of NACA 0012 aerofoil at $R e=50,000$ is shown in figure 1; experimental [2] and CFD results from different sources $[4,8]$ are also plotted for comparison. $T u$, in the present computations, was set to $0.1 \%$ in conformance with the experimental set-up of Ohtake et al [2]. $C_{l}$ agrees well with the experimental results till about $\alpha=8^{\circ}$, indicating a validation of the present computational procedure. The difficulties in achieving good agreement between experiments and computations at low to moderate $R e$ are discussed in [3], while in particular, the disagreement observed at high angles of attack is discussed in [9]. Several interesting observations can be made from the lift characteristic curve: non-linear lift curve, negative $C_{l}$ at low angles of attack $\left(\alpha \leq 1^{\circ}\right)$, recovery from negative $C_{l}$ and sudden change of slope in $C_{l}-\alpha$ curve at $\alpha=3^{\circ}$ and $9^{\circ}$. Physical explanations of these observations are detailed in the subsequent subsections.

\subsection{Negative lift}

Negative lift on a symmetrical aerofoil at a positive $\alpha$ appears to be counter-intuitive even though there is no law or principle that prohibits it. These intuitions probably are picked up from the potential thin aerofoil theory, where we have a remarkable result $C_{l}=2 \pi \alpha$, without giving any scope for doubting near $\alpha=0^{\circ}$ due to the constant slope.

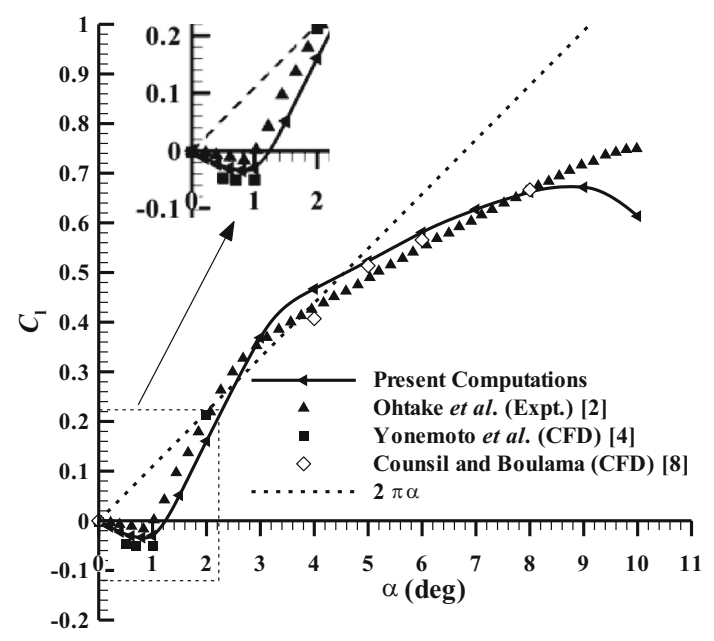

Figure 1. Variation of $C_{l}$ at $R e=50,000$.
Particle dynamics, like Newton's corpuscular theory, even though not valid in a field since the continuity constraint has to be satisfied, also supports such prejudice: a flat plate at a positive $\alpha$ should provide positive lift due to particle collisions and consequent momentum transfer. However, the effect of camber in the thin aerofoil theory may be worth recalling here. Camber leads to positive lift, $C_{l}$ being linear in camber. In the present low-Re high viscous flow at small positive angles of attack, the separated flow over the lower surface turns around the trailing edge (T.E.) and flows into the separated region over the upper surface, resulting in the violation of the Kutta condition, as can be seen in figure 2.

The presence of such a separated region and also the differential growth of the boundary layer displacement thickness on either side add to the equivalent thickness distribution to the aerofoil, leading to an effective negative camber, which in turn results in a net negative circulation. For such cases, the pressure on the upper surface is greater than the pressure on the lower surface for a significant part of the aerofoil chord, leading to the generation of net negative lift; see figure 3 .

Selig et al [10], in their experiments on symmetrical aerofoils at low $R e$, inferred that the change in effective shape of the aerofoil led to insignificant values of $C_{l}$ at $\alpha$ close to $0^{\circ} . C_{l}$ did not quite reach a negative value in that study, whereas in the experiments of [2] and [3] the values of $C_{l}$ are not just negligible but are negative as supported by

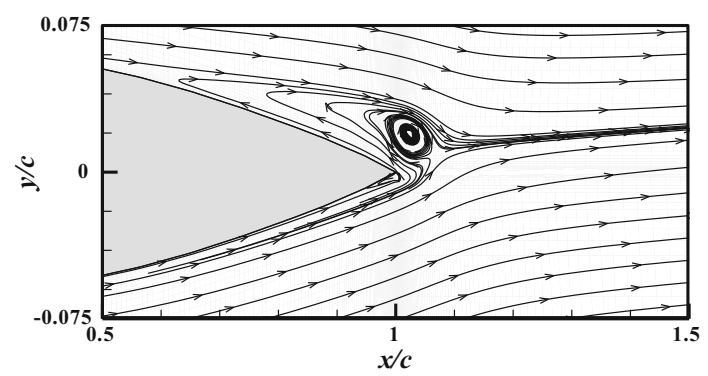

Figure 2. Streamlines close to trailing edge; $\alpha=1^{\circ}$ and $C_{l}=-0.0299$.

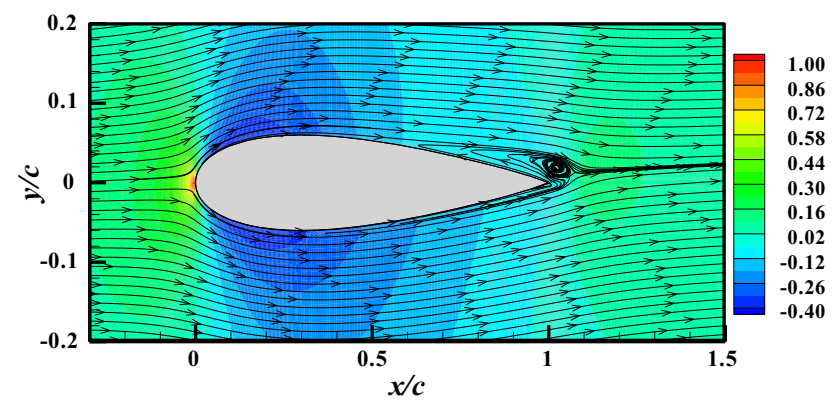

Figure 3. Streamlines and contours of pressure coefficient over the aerofoil; $\alpha=1^{\circ}$. 
Table 1. Summary of separation $\left(x_{s}\right)$, transition $\left(x_{t}\right)$ and reattachment $\left(x_{r}\right)$ locations on the upper surface of the aerofoil; Re $=50,000$ and $T u=0.1 \%$.

\begin{tabular}{|c|c|c|c|c|c|c|c|c|c|c|}
\hline \multirow[b]{2}{*}{ Regime } & \multirow{2}{*}{$\begin{array}{l}\alpha \\
\left({ }^{\circ}\right)\end{array}$} & \multirow[b]{2}{*}{$C_{l}$} & \multirow[b]{2}{*}{ Flow regime } & \multicolumn{4}{|c|}{ Laminar separation bubble } & \multicolumn{3}{|c|}{$\begin{array}{l}\text { Trailing edge } \\
\text { recirculation }\end{array}$} \\
\hline & & & & $x_{s} / c$ & $x_{t} / c$ & $x_{r} / c$ & $L_{L S B} / c$ & $x_{s T} / c$ & $x_{r T} / c$ & $L_{T E R} / c$ \\
\hline \multirow[t]{6}{*}{1} & 0 & 0.0000 & \multirow{6}{*}{$\begin{array}{l}\text { Laminar separation without transition and without } \\
\text { reattachment }\end{array}$} & 0.67 & - & \multirow{6}{*}{\multicolumn{2}{|c|}{$\begin{array}{c}\text { Flow does } \\
\text { not } \\
\text { reattach }\end{array}$}} & \multirow{6}{*}{\multicolumn{3}{|c|}{$\begin{array}{c}\text { Turbulent separation } \\
\text { was not observed }\end{array}$}} \\
\hline & 0.2 & -0.0120 & & 0.63 & - & & & & & \\
\hline & 0.4 & -0.0225 & & 0.60 & - & & & & & \\
\hline & 0.6 & -0.0305 & & 0.58 & - & & & & & \\
\hline & 0.8 & -0.0338 & & 0.55 & - & & & & & \\
\hline & 1 & -0.0299 & & 0.52 & - & & & & & \\
\hline \multirow[t]{3}{*}{2} & 1.5 & 0.0515 & \multirow{3}{*}{$\begin{array}{l}\text { Laminar separation with transition and without } \\
\text { reattachment }\end{array}$} & 0.48 & 0.98 & & & & & \\
\hline & 2 & 0.1605 & & 0.43 & 0.95 & & & & & \\
\hline & 3 & 0.4007 & & 0.32 & 0.81 & & & & & \\
\hline \multirow[t]{2}{*}{3} & 4 & 0.4661 & \multirow{2}{*}{$\begin{array}{l}\text { Laminar separation with transition and with } \\
\text { reattachment (LSB) }\end{array}$} & 0.23 & 0.67 & 0.80 & 0.57 & & & \\
\hline & 5 & 0.5200 & & 0.14 & 0.53 & 0.66 & 0.51 & & & \\
\hline \multirow[t]{3}{*}{4} & 6 & 0.5747 & \multirow[t]{3}{*}{ LSB with Trailing Edge Recirculation (TER) } & 0.07 & 0.38 & 0.50 & 0.42 & 0.97 & 1 & 0.03 \\
\hline & 7 & 0.6171 & & 0.04 & 0.29 & 0.38 & 0.34 & 0.93 & 1 & 0.07 \\
\hline & 8 & 0.6482 & & 0.03 & 0.22 & 0.34 & 0.31 & 0.82 & 1 & 0.18 \\
\hline \multirow[t]{2}{*}{5} & 9 & 0.6566 & \multirow[t]{2}{*}{ Merging of LSB and TER } & 0.02 & 0.18 & - & - & - & 1 & - \\
\hline & 10 & 0.6052 & & 0.02 & 0.17 & - & - & - & 1 & - \\
\hline
\end{tabular}

the present study. It is equivalent to the usual $C_{l}-\alpha$ curve passing through the origin being shifted to the right due to an effective negative camber, giving a narrow range of $\alpha$ where $C_{l}$ is negative. However, symmetry of the aerofoil requires that the origin has to be a valid point on the $C_{l}-\alpha$ curve, thus leading to the shape of the $C_{l}-\alpha$ curve as shown in figure 1. With this understanding it should be possible to predict the role of various parameters involved. Thus, an increase in $\alpha, R e$ and freestream turbulence or flow transition to turbulence should counteract this interesting and counter-intuitive behaviour. The role of aerofoil thickness distribution is expected to be complicated. As regards the drag there is no such chance of change of sign as dictated by the momentum or energy principles.

\subsection{Effect of angle of attack}

Based on the separation, transition and reattachment locations, we identify five distinct flow regimes over the aerofoil upper surface, see table 1 . As $\alpha$ increases, the separation location on the upper surface moves towards the L.E. whereas on the lower surface it moves towards the T.E. The contours of intermittency revealed that flow transition to turbulence did not occur over the aerofoil upper surface for $\alpha \leq 1^{\circ}$, where negative $C_{l}$ was observed; see figure $4 \mathrm{a}$. On the other hand, for $\alpha>1^{\circ}$ flow transition to turbulence occurs over the aerofoil surface. It may be observed that the pressure on the lower surface is greater than the pressure on the upper surface along the entire chord for $\alpha=2^{\circ}$ and $3^{\circ}$, leading to recovery from negative lift, and eventual positive lift; see figure $4 \mathrm{~b}$. The core of the upper surface recirculation region is found to occur downstream of the aerofoil for all cases with negative $C_{l}$ $\left(\alpha \leq 1^{\circ}\right)$, whereas it occurs over the aerofoil surface for $\alpha>1^{\circ}$. Streamlines over the aerofoil at $\alpha=1.5^{\circ}, 2^{\circ}$ and $3^{\circ}$ reveal that the Kutta condition is found to be violated even for these cases where positive $C_{l}$ is observed.

For $\alpha=1.5^{\circ}, 2^{\circ}$ and $3^{\circ}$, the flow transitions to turbulence, but the turbulent boundary layer on the upper surface does not reattach. However, at $\alpha=4^{\circ}$, the turbulent boundary layer reattaches, resulting in the formation of LSB on the upper surface, while a recirculation region (laminar separation and laminar reattachment) is observed on the lower surface close to the T.E. Further increase in $\alpha$ results in shorter LSB on the upper surface which moves towards the L.E. while the recirculation region on the lower surface disappears. Kutta condition was found to be satisfied for $\alpha \geq 4^{\circ}$. Also, at $\alpha=6^{\circ}$, apart from the presence of LSB, turbulent separation is observed at the rear part of the aerofoil upper surface which reattaches at the T.E. As $\alpha$ increases, the turbulent separation location moves upstream while the reattachment location remains insensitive to $\alpha$. At higher angles of attack $\left(\alpha \geq 9^{\circ}\right)$, the L.E. LSB merges with the Trailing Edge Recirculation (TER) region, resulting in the formation of a large separated region that spreads almost over the entire chord length. It is in this range of $\alpha$ where the results from the present simulations differ significantly from the experimental results (see figure 1).

Among various flow regimes observed, negative values of lift coefficient are found to occur only at low angles of attack, which corresponds to laminar separation without flow transition and without reattachment. In the subsequent sections we address various factors that influence this phenomenon of negative lift and its recovery. 


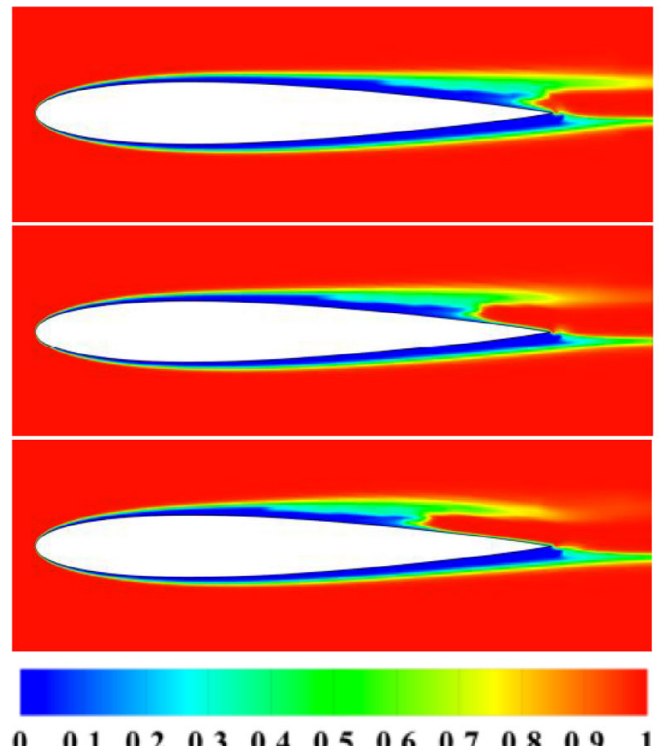

(a)

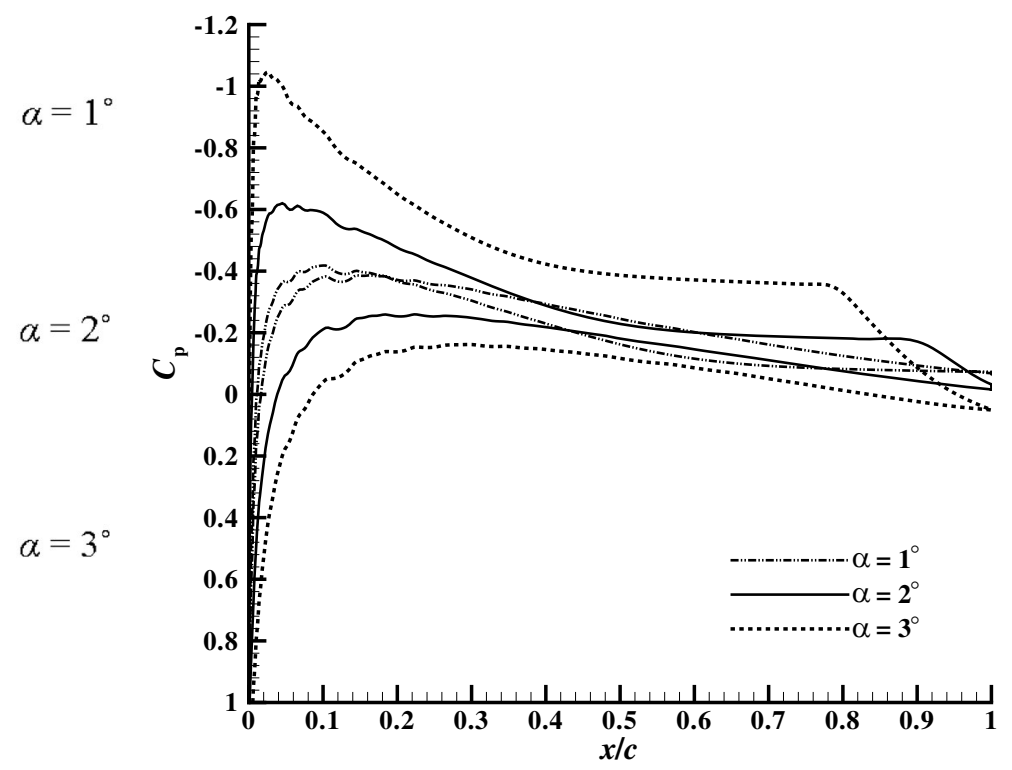

(b)

Figure 4. (a) Contours of intermittency and (b) pressure distribution at various $\alpha ; R e=50,000$.

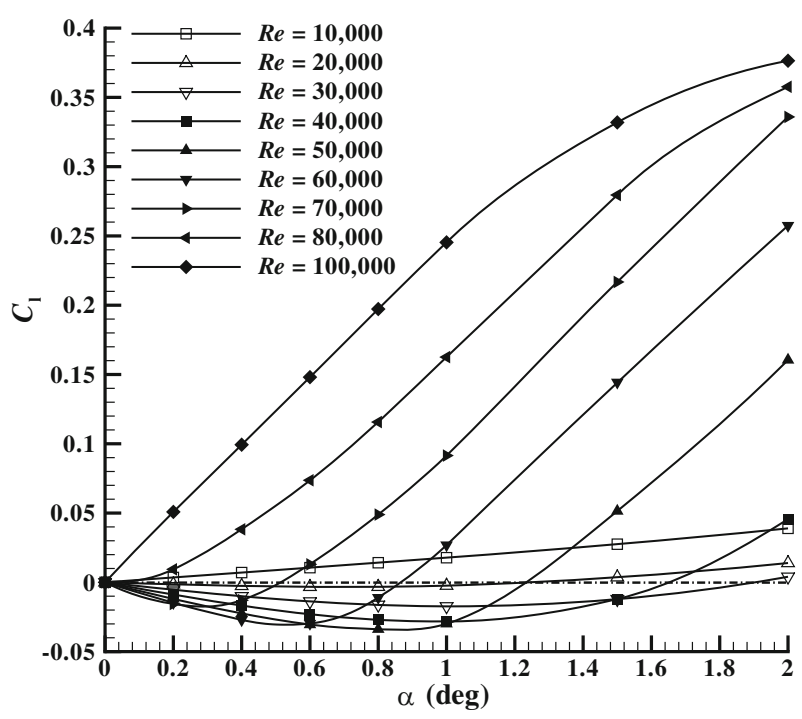

Figure 5. Effect of $R e$ on negative lift; $T u=0.1 \%$.

\subsection{Effect of Reynolds number}

To study the effect of $R e$ on negative lift, computations were carried out in the $R e$ range of 10,000-100,000 for several values of $\alpha$ between $0^{\circ}$ and $2^{\circ}$. Tu was maintained at $0.1 \%$. Based on the lift characteristics, we identify three different $R e$ regimes. In the $R e$ range of 20,000-30,000, as $R e$ increases, the range of $\alpha$ with negative $C_{l}$ increases, whereas in the $R e$ range of 40,000-75,000, the range of $\alpha$ with negative $C_{l}$ decreases with increasing $R e$; see figure 5 . A further increase in $\operatorname{Re}(\operatorname{Re}>75,000)$ resulted in the disappearance of negative lift. The lift curve slope $C_{l, \alpha}$

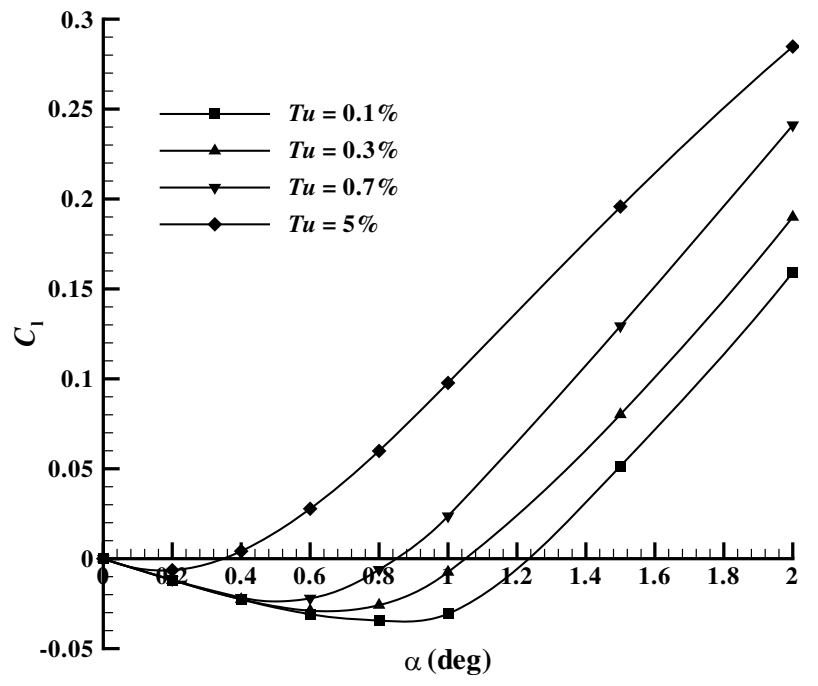

Figure 6. Effect of $T u$ on negative lift; $R e=50,000$.

evaluated at $\alpha=0^{\circ}$ from the present study agrees well with the results available in [2] in the entire $R e$ range (not shown here for brevity). $C_{l, \alpha}\left(\alpha=0^{\circ}\right)$ starts to become negative in the $R e$ range of $10,000-20,000$ and it recovers positive slope in the $R e$ range of 75,000-80,000.

\subsection{Effect of freestream turbulence}

It is well known that increasing the freestream $T u$ results in an early transition; hence higher turbulence intensities can be expected to eliminate the occurrence of negative lift. To verify this assertion, computations were carried out at 


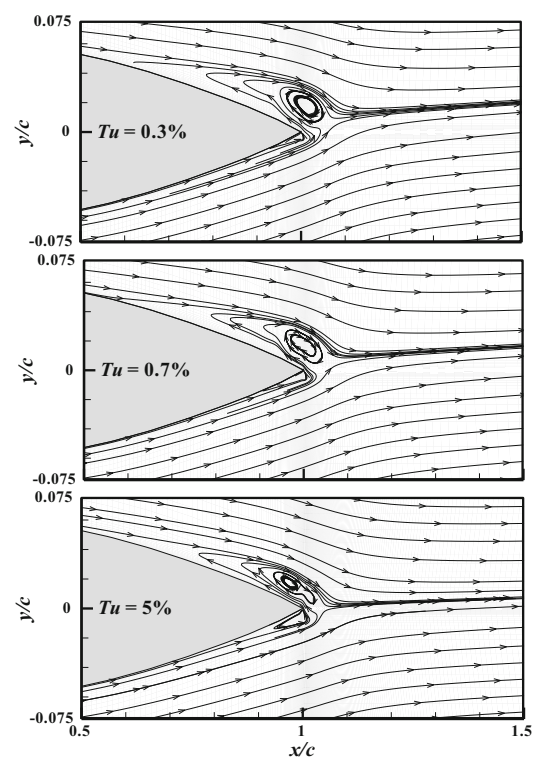

(a)

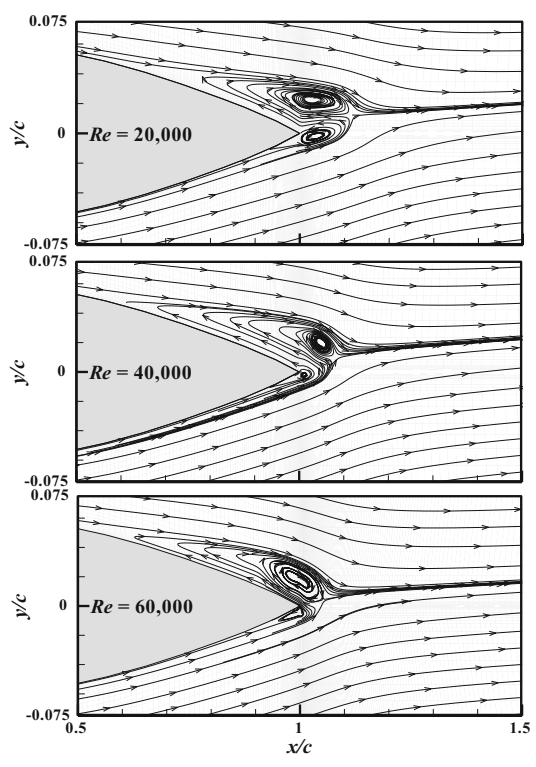

(b)
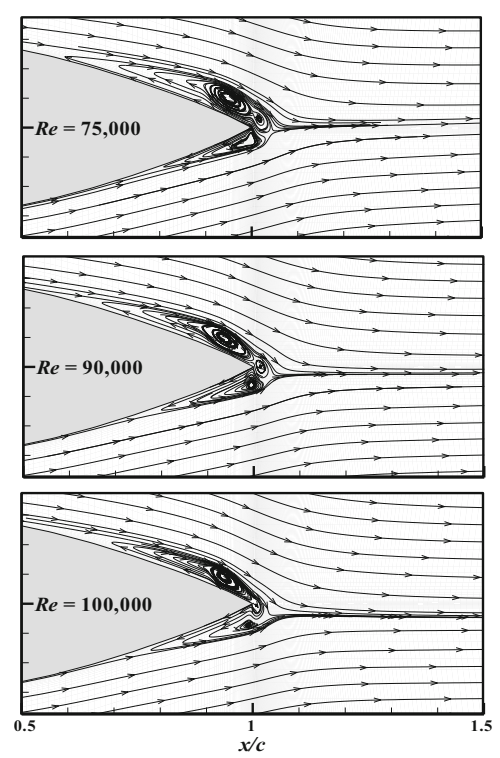

Figure 7. Streamlines close to T.E. at $\alpha=1^{\circ}$ : (a) effect of $T u$ at $\operatorname{Re}=50,000$ and (b) effect of $\operatorname{Re}$ at $T u=0.1 \%$.

$R e=50,000$ for several values of $\alpha$ ranging from $0^{\circ}$ to $2^{\circ}$ at higher turbulence intensities of $0.3 \%, 0.7 \%$ and $5 \%$. The results are presented in figure 6 . It can be seen that despite high values of $T u$, which promotes early transition, negative values of $C_{l}$ are still observed. However, the range of $\alpha$ at which such a phenomenon occurred and the magnitude of negative $C_{l}$ are curtailed.

The effect of $T u$ on streamline pattern at $\alpha=1^{\circ}$ and $R e=50,000$ is shown in figure 7a, while the effect of $R e$ on streamlines over the aerofoil at $\alpha=1^{\circ}$ and $T u=0.1 \%$ is shown in figure $7 \mathrm{~b}$. A careful interpretation of flow features revealed some similarities between the effects of increasing $R e$ and that of increasing freestream $T u$ on the recovery from the negative values of lift coefficient. As $R e$ and $T u$ increase, the transition location initially observed in the wake at a downstream point gradually shifts closer to the T.E. and eventually occurs over the aerofoil upper surface. An important insight that can be inferred from these observations is that, for the recovery from negative lift, the transition location does not necessarily have to occur over the aerofoil upper surface but can occur downstream of the aerofoil but sufficiently close to the T.E. such that the core of the upper surface recirculation region sits over the aerofoil surface. It is also observed that, as $R e$ and $T u$ increase, the thickness of the separated region on the upper surface decreases while on the lower surface it increases.

Yonemoto et al [4] postulate that the disappearance of the reverse flow region around the T.E. is the reason for the recovery from the negative lift with increasing Reynolds numbers. However, in the present study it is observed that the reverse flow region covering the T.E. is present even for cases where a positive value of $C_{l}$ is observed; for instance, see figure $7 \mathrm{~b}(\operatorname{Re} \geq 60,000)$. It may be worth mentioning that the Kutta condition was found to be violated for all the cases with negative values of lift coefficient; however, even for some cases with values of positive $C_{l}$ the Kutta condition was found to be violated.

\section{Conclusion}

The unusual lift characteristics exhibited by symmetrical aerofoils at low Reynolds numbers was studied by computationally investigating the flow field around NACA 0012 aerofoil. There exists a narrow range of $\alpha$ close to $0^{\circ}$ where the lift coefficient is negative for positive $\alpha$. Five flow regimes have been identified, of which laminar separation case without flow transition to turbulence and without reattachment is the flow regime associated with this counter-intuitive behaviour of the $C_{l}-\alpha$ dependence. The Kutta condition was found to be violated for all the cases with negative values of lift coefficient. However, it was also observed that not all cases violating the Kutta condition resulted in negative lift. The shape of the separated region, along with the differential growth of boundary layer displacement thickness on the upper and lower surfaces together, introduces an effective negative camber, resulting in a net negative circulation around the aerofoil, thereby generating negative lift. Further, it is concluded that, increasing the Reynolds number or the angle of attack or the freestream turbulence results in an early transition, which in turn reduces the influence of the recirculation region on the pressure distribution over the aerofoil surface, thereby recovering positive lift. 


\section{References}

[1] Mueller T J and Batill S M 1982 Experimental studies of separation on a two-dimensional airfoil at low Reynolds numbers. AIAA J. 20: 457-463

[2] Ohtake T, Nakae Y and Motohashi T 2007 Nonlinearity of the aerodynamic characteristics of NACA0012 aerofoil at low Reynolds numbers. J. Jpn. Soc. Aeronaut. Space Sci. 55: 439-445

[3] Tank J, Smith L and Spedding G R 2017 On the possibility (or lack thereof) of agreement between experiment and computation of flows over wings at moderate Reynolds number. Interface Focus 7: 1-15

[4] Yonemoto K, Takato K, Ochi H and Fujie S 2008 Kutta condition violation in two-dimensional NACA0012 airfoil at low Reynolds number. AIAA Paper 2008-6399

[5] Lee D, Nonomura T, Oyama A and Fujii K 2014 Comparison of numerical methods evaluating airfoil aerodynamic characteristics at low Reynolds number. J. Aircr. 52: 296-306

[6] Menter F R, Langtry R B, Likki S R, Suzen Y B, Huang P G and Völker S 2006 A correlation-based transition model using local variables_ part I: model formulation. J. Turbomach. 128: 413-422

[7] Langtry R B, Menter F R, Likki S R, Suzen Y B, Huang P G and Völker S 2006 A correlation-based transition model using local variables-part II: test cases and industrial applications. J. Turbomach. 128: 423-434

[8] Counsil J N and Boulama K G 2013 Low-Reynolds-number aerodynamic performances of the NACA 0012 and SeligDonovan 7003 airfoils. J. Aircr. 50: 204-216

[9] Chen Z J, Qin N and Nowakowski A F 2013 Three-dimensional laminar-separation bubble on a cambered thin wing at low Reynolds numbers. J. Aircr. 50: 152-163

[10] Selig M S, Guglielmo J F, Broern A P and Giguere P 1996 Experiments on airfoils at low Reynolds numbers. AIAA Paper 96-0062 